\title{
A Quasi-Classical Model of Intermediate Velocity Particle Production in Asymmetric Heavy Ion Reactions
}

\author{
A. Chernomoretz ${ }^{1}$, L. Gingras ${ }^{2}$, Y. Larochelle ${ }^{2 *}$, L. Beaulieu ${ }^{2}$, R. Roy ${ }^{2}$, C. St-Pierre ${ }^{2}$, C.O.Dorso ${ }^{1}$ \\ ${ }^{1}$ Departamento de Fisica, Universidad de Buenos Aires, Buenos Aires, Argentina. \\ ${ }^{2}$ Laboratoire de Physique Nucléaire, Département de Physique, Université Laval, Québec, QC, Canada G1K $7 P 4$.
}

(October 31, 2018)

\begin{abstract}
The particle emission at intermediate velocities in mass asymmetric reactions is studied within the framework of classical molecular dynamics. Two reactions in the Fermi energy domain were modeled, ${ }^{58} \mathrm{Ni}+\mathrm{C}$ and ${ }^{58} \mathrm{Ni}+\mathrm{Au}$ at 34.5 $\mathrm{MeV} /$ nucleon. The availability of microscopic correlations at all times allowed a detailed study of the fragment formation process. Special attention was paid to the physical origin of fragments and emission timescales, which allowed us to disentangle the different processes involved in the mid-rapidity particle production. Consequently, a clear distinction between a prompt pre-equilibrium emission and a delayed aligned asymmetric breakup of the heavier partner of the reaction was achieved.
\end{abstract}

PACS number(s): 25.70 -z, 25.70.Mn, 25.70.Pq, 02.70.Ns

\section{INTRODUCTION}

Heavy ion reactions in the Fermi energy domain (20 $100 \mathrm{MeV} /$ nucleon) has been an intense field of research during the last decade. In particular, a lot of attention was paid to the strong emission of intermediate mass fragments (IMF) observed in the mid-rapidity range. [2 10]. Lying in rapidity space between the contributions of the projectile-like and target-like fragments, this complex emission pattern can not be explained as a result of a simple statistical emission of the two excited participant nuclei. This fact suggests that it should be considered as a non-trivial phenomenon in which the interplay of the two-body interactions and the nuclear mean field plays a crucial role.

There is not yet a uniform agreement about the possible mechanisms responsible for this IMF overproduction at mid-rapidity. Recently, a statistical approach to the problem was developed [11], and the claim that the inclusion of Coulombian interactions in the statistical picture could explain this behavior was made. On the contrary, some authors adopted a dynamical description to analyze the same process. For example, a Landau-Vlasov semiclassical transport model was used to analyze the

\footnotetext{
*Present address: Joint Institute for Heavy Ion Research, Holifield Radioactive Ion Beam Facility, Oak Ridge, Tennessee 37831-6374.
}

presence 7 and the temporal behavior [12 of the particle emission process that populates the mid-rapidity range. Neck instabilities, dynamical fluctuations, and particle production at mid-velocities were also studied using the quantum molecular dynamics (QMD) model in semi-peripheral [13], and central heavy ion collisions [14. In addition, in a recent contribution, 15], two mechanisms of IMF production were suggested (neck, and fission-like surface emission), and two different models were introduced in order to describe them.

The aim of this paper is to present a simple model that provides a description of the IMF production process at mid-velocities. In particular, we focus our attention on two mass asymmetric reactions of relatively small systems, ${ }^{58} \mathrm{Ni}+\mathrm{C}$ and ${ }^{58} \mathrm{Ni}+\mathrm{Au}$ at $34.5 \mathrm{MeV} /$ nucleon. The motivation to study these systems is two-fold. On one hand, it allows us to resolve the controversy about fast overlap emission processes and slow surface deformation breakup. On the other, our time-based analysis supports new experimental observations of alignment and proximity effects in neck breakup of mass asymmetric collision partners 16.

The reactions are studied within a quasi-classical approach using molecular dynamics techniques. Out of various methods used to study heavy ion reactions, the implemented MD model can describe changes of phase, hydrodynamic flow, and non-equilibrium dynamics without adjustable parameters. All order nucleon-nucleon correlations to form fragments and neck-like structures are intrinsically incorporated in the dynamics. Of course, the price one has to pay using such a simplified quasiclassical description is that quantitative agreement with experiments can not be taken for granted. Nevertheless, despite its simplicity, we found it a great tool to probe new physical effects and to propose a meaningful scenario for different origins of MR particles (see [16]).

Several kinematical features of the experimental data are well reproduced. In addition, the availability of correlations of all orders at all times allowed us to study in detail the time evolution of the fragment formation process of IMF's and to identify different mechanisms of midrapidity particle production within a unified description. In this way, three time-scales could be differentiated: a violent pre-equilibrium stage, a delayed aligne emission stage, and a "statistical" evaporation stage.

In section II, we briefly describe the experimental setup. The description of the nuclear interactions model and the used fragment recognition algorithm are given 
in sections III and IV respectively. The obtained results and their posterior analysis are included in sections $\mathrm{V}$ and VI. Finally, conclusions are drawn in section VII.

\section{EXPERIMENTAL DATA}

The two mass asymmetric reactions studied in this contribution were performed at the coupled Tandem and Super-Conducting Cyclotron accelerators of AECL at Chalk River. A beam of ${ }^{58} \mathrm{Ni}$ accelerated at 34.5 $\mathrm{MeV} /$ nucleon bombarded alternatively a $2.4 \mathrm{mg} / \mathrm{cm}^{2}$ carbon target and a $2.7 \mathrm{mg} / \mathrm{cm}^{2}$ gold target. Charged particles issued from these reactions were detected in the CRL-Laval $4 \pi$ array constituted by 144 detectors set in ten rings concentric to the beam axis and covering polar angles between $3.3^{\circ}$ and $140^{\circ}$. See [17] and references therein for more information on detectors and energy calibration. In the present work, we will consider events selected in the off-line analysis by a total detected charge of at least 24 and 34 (fully detected) units for the ${ }^{58} \mathrm{Ni}+\mathrm{Au}$, and ${ }^{58} \mathrm{Ni}+\mathrm{C}$ systems respectively.

\section{THE MODEL}

In order to get information on the time formation and the kinematical characteristics of the intermediate velocity material produced in these mass asymmetric heavy ion reactions, we relied on molecular dynamics simulations. The model used in this work was originally introduced by Lenk et al. to test the accuracy of the VlasovNordheim approximation 18. It is a classical molecular dynamics (CMD) model with a spherical two-body interaction potential in which Coulombian interactions are taken into account, while the nuclear flavor is provided by the following interaction terms:

$$
\begin{aligned}
& V_{n p}=V_{r}\left[\frac{e^{-\mu_{r} r}}{r}-\frac{e^{-\mu_{r} r_{c}}}{r_{c}}\right]-V_{a}\left[\frac{e^{-\mu_{a} r}}{r}-\frac{e^{-\mu_{a} r_{c}}}{r_{c}}\right] \\
& V_{n n}=V_{0}\left[\frac{e^{-\mu_{0} r}}{r}-\frac{e^{-\mu_{0} r_{c}}}{r_{c}}\right]
\end{aligned}
$$

$V_{n p}$ and $V_{n n}$ stand for neutron-proton and identical nucleons interactions respectively, and $r_{c}=5.4 \mathrm{fm}$ is a cutoff radius. We used a set of parameters (M-set in Ref. [18]), that gives an equation of state with a compressibility around $250 \mathrm{MeV}$, an equilibrium density of $\rho_{0}=0.16 \mathrm{fm}^{-3}$, and $E\left(\rho_{0}\right)=-16 \mathrm{MeVA}$. Due to the particular choice of parameters, this model provides a realistic nucleon-nucleon cross section and it has been used to reproduce reasonably well several features of experimental data on heavy ion collisions (see 18 21] and references therein)

The nuclei used in our computational experiments have been built with the right number of protons and neutrons while the corresponding ground-states were obtained by molecular dynamic techniques, i.e. by a cooling procedure that starts with a confined and rather excited nucleus, and ends with a self-contained state at reasonable binding energies. Before each collision a random relative projectile-target orientation was chosen, and afterwards the projectile's center of mass velocity was boosted to the desired energy. The set of equation of motion is integrated up to a final time of $t=2000 \mathrm{fm} / \mathrm{c}$ with a standard velocity-Verlet algorithm 22], taking $t_{\text {int }}=0.02 \mathrm{fm} / \mathrm{c}$ as the integration time step, achieving an energy conservation of $0.01 \%$. Under this scheme, we have analyzed 8400 collisional events for the ${ }^{58} \mathrm{Ni}+{ }^{12} \mathrm{C}$ at $34.5 \mathrm{MeV} /$ nucleon reaction, for a wide range of impact parameter values $(b \leq 4.5 \mathrm{fm})$, and 3400 events for the ${ }^{58} \mathrm{Ni}+{ }^{197} \mathrm{Au}$ at 34.5 $\mathrm{MeV} /$ nucleon case $(5 \mathrm{fm} \leq b \leq 8 \mathrm{fm})$.

\section{FRAGMENT RECOGNITION}

In order to explore the nucleon-nucleon correlations that give rise to the fragment formation process, we have adopted a definition of cluster compatible with the clusterization algorithm known in the literature as the minimum spanning tree in energy space (MSTE) 23]. Under the MSTE scheme a given set of particles $i, j, \ldots, k$ belongs to the same cluster $C_{a}$ if:

$$
\forall i \in C_{a}, \exists j \in C_{a} / e_{i j} \leq 0
$$

where $e_{i j}=V\left(r_{i j}\right)+\left(\mathbf{p}_{i}-\mathbf{p}_{j}\right)^{2} / 4 \mu$, and $\mu$ is the reduced mass of the pair $\{i, j\}$. This clusterization method searches for correlated structures in $\mathbf{q}$ space (first term in the definition of $e_{i j}$ ), with the additional possibility of avoiding certain particle incorporation to a given cluster regarding at the relative momenta of particle pairs (second term of $e_{i j}$ ). In this way the conformation of the MSTE partitions reflects certain degree of correlation in q-p space. Due to this feature, the MSTE becomes very useful in the recognition process of promptly emitted particles during the pre-equilibrium stage of the reaction.

The availability of correlations of all-orders at all times allows us to gain a lot of insight about the presence and origin of mid-velocity sources. For instance, right after the most violent stage of the collision, an MSTE cluster recognition step enables the tagging of particles as belonging to the quasi-projectile (QP), the quasi-target (QT), or as free particles (FP) not $\mathbf{q}-\mathbf{p}$ correlated neither to the QT nor the QP at tagging time $\left(t_{\text {tag }}\right)$.

The value of $t_{\text {tag }}$ is determined on an event by event basis. Analyzing the temporal behavior of the size of the two biggest clusters, $t_{t a g}$ is associated with the time at which the mass number of these fragments attains stability for the first time after the fragmentation process has started 24]. Figure 1illustrates this last point. It shows a typical $t_{t a g}$ determination for a single ${ }^{58} \mathrm{Ni}+{ }^{197} \mathrm{Au}$ reaction with an impact parameter value of $b=6 \mathrm{fm}$. 


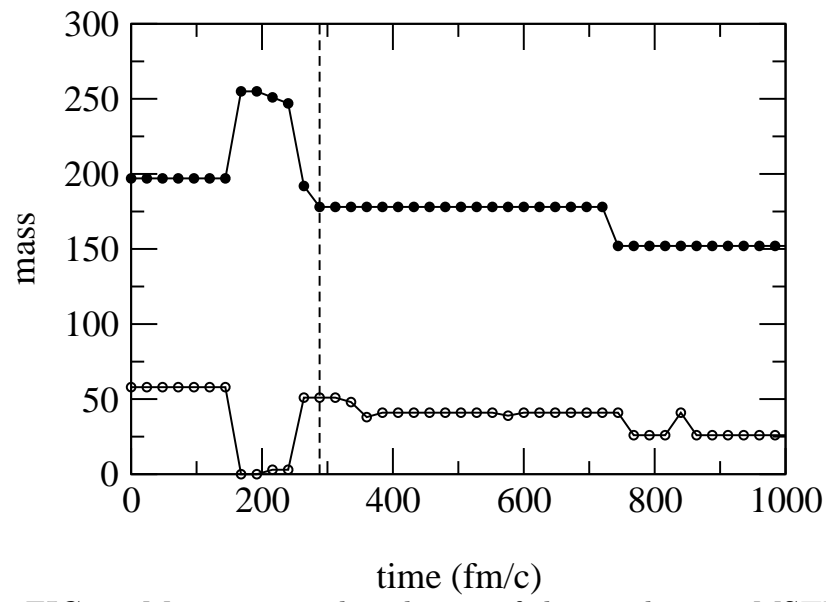

FIG. 1. Mass temporal evolution of the two biggest MSTE clusters for a typical ${ }^{58} N i+{ }^{197} A u$ reaction with an impact parameter value of $b=6 \mathrm{fm}$. The dashed line signals the estimated $t_{\text {tag }}$

It is then clear that $t_{\text {tag }}$ signals the entrance into a new and less violent deexcitation regime with particles $\mathrm{QP}(\mathrm{QT})$-tagged being well correlated in configuration space right after the highly collisional stage.

In addition, the microscopic description allows to easily calculate the time $\left(t_{e}\right)$ at which every fragment has actually been emitted by simply tracing back the dynamical evolution of the reaction. Since each asymptotic detected cluster is emitted at different stages of the reaction (a cluster is considered as a whole when most of its nucleon constituents get well correlated velocities and positions, differentiating themselves from other fragments) it is possible to extract useful information correlating this emission time characterization with other kinematical variables.

\section{RESULTS}

\section{A. Reliability of the simulations}

In order to compare the numerical simulations with the experimental data the following prescription was adopted to estimate an observable $\left(b_{\exp }\right)$ related to the reaction impact parameter (see [16], [25]):

$$
b_{\text {exp }}=r_{0}\left(A_{P}^{1 / 3}+A_{T}^{1 / 3}\right) \frac{\Pi_{\|}^{c m}}{P_{P}^{c m}}
$$

where $\Pi_{\|}^{c m}$ is the total parallel momentum of all charged particles in the forward velocity hemisphere of the center of mass $(\mathrm{CM})$ reference frame, $A_{P}, A_{T}$ and $P_{P}^{c m}$ are respectively the projectile mass number, target mass number and projectile CM momentum. We took $r_{0}=1.2 \mathrm{fm}$.
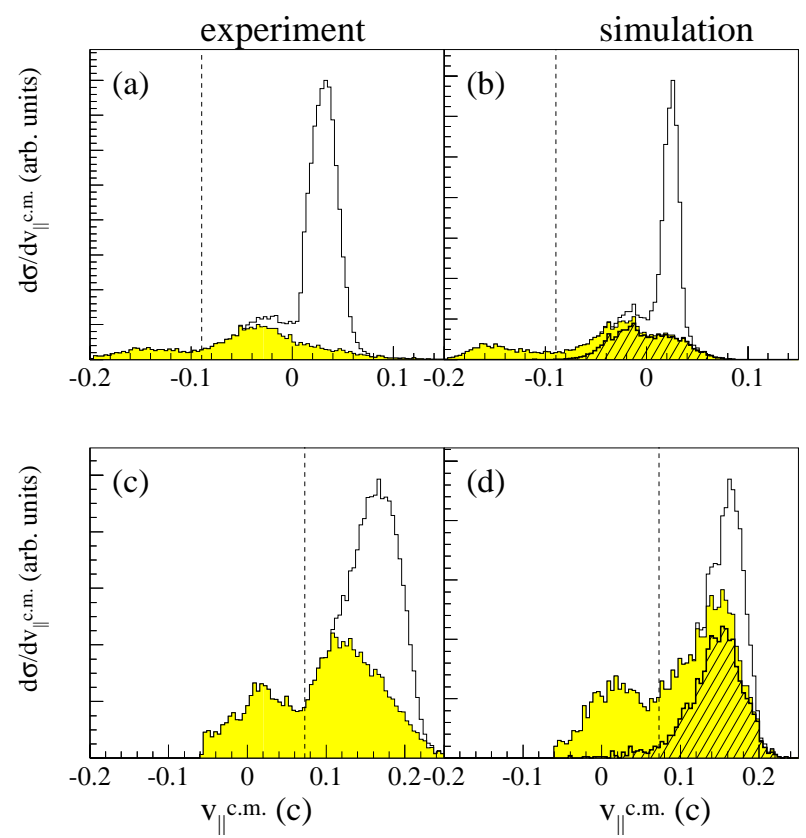

FIG. 2. Normalized parallel velocity distribution, calculated in the center of mass system for $z \geq 3$ particles, corresponding to mid-peripheral and peripheral ${ }^{58} \mathrm{Ni}+\mathrm{C}$ and ${ }^{58} \mathrm{Ni}+\mathrm{Au}$ reactions are shown in upper and lower panels respectively. Vertical dashed lines were drawn at nucleon-nucleon velocity value. Figures (a) and (c) show experimental results, whereas (b) and (d) show simulated ones. In each panel the IMF contribution is shadowed, whereas QP tagged IMF's are stripped. See text for details.

In panels (a) and (b) of figure 2 we show the normalized parallel velocity distribution $\left(v_{\|}^{c m}\right)$, in the CM reference frame, of the $z \geq 3$ collision charged products for ${ }^{58} \mathrm{Ni}+{ }^{12} \mathrm{C}$ peripheral and mid-peripheral $\left(b_{\text {exp }} \geq 2.5\right.$ $\mathrm{fm})$ reactions. Experimental data is shown in the first panel and simulation results in the second one. The IMF contribution $(3 \leq z \leq 7)$ was shadowed and quasi projectile assigned IMF were stripped . (The $4 \pi$ detector coverage and the complete detected charge condition allow us to establish, in this case, a meaningful comparison between the experimental data and unfiltered simulated reactions ).

It can be seen that general features are well reproduced by the simulation. Aside from PLF and TLF velocity signatures, an intermediate bump peaked in between $v_{n n}$ and PLF velocity can be recognized for both, experimental and simulated data. Nevertheless, it seems that the CMD model overestimates slightly the amount of IMF's coming from the QP in the higher velocity region of $v_{\|}^{c m} \sim 0.03 c$.

The corresponding normalized parallel velocity distributions for ${ }^{58} \mathrm{Ni}+\mathrm{Au}$ mid-peripheral and peripheral reactions $\left(6 \mathrm{fm}<b_{\text {exp }}\right)$, are shown in panels $(\mathrm{c})$ and $(\mathrm{d})$ of figure 2. In this case, simulated data were filtered using a software implementation of the detector. Despite the completely different entrance channel of this reaction, 
and the different effect played by the detection thresholds of the apparatus, similar conclusions can be drawn for this reaction: general features of the distribution are well reproduced by the model, but an overproduction of IMF coming from the QP (centered at $v_{\|}^{c m} \sim 0.15 c$ ) can also be recognized in panel (d).

We believe that this happens because our simplified model fails to accurately reproduce secondary deexcitation processes at a quantitative level. In particular, the relative amplitude of LCP's and IMF's production in the QP exit channel is not well reproduced because the quasiclassical approach can not adequately handle the relevant degrees of freedom involved in the slow nuclear deexcitation processes.

Another point that is worth noting is that, as the difference between the mid-rapidity bump (on the QP side) and $v_{n n}$ velocities seems to be similar for both reactions (panels (a), and (c)), one can conclude that the deexcitation process of the QP should be the same. Within our model, we can not rule out the possibility that in both reactions the QP proceed thru similar IMF production mechanisms. In particular, it seems possible that the process of delayed asymmetric breakup (see Sec.VB 2 ) is there in both cases. However, along the present contribution, plausibility arguments will be given supporting the idea that this happens with different probabilities in each case.

From the preceeding analysis, it can be concluded that, even if a quantitative description of the process seems to be questionable, the use of our CMD model can throw some light upon the problem of mid rapidity production. As will be shown, despite its simplicity, the model is flexible enough to allow the presence of qualitatively different mechanisms of particle production that can be integrated in a unified description of the process. This can be used to settle a meaningful scenario within which experimental data can be studied.

\section{B. CMD Description}

\section{Source-origin analysis}

The microscopic description of the reaction allows us to associate two parameters to each asymptotic charged particle: its emission time $\left(t_{e}\right)$, and its early physical origin, i.e if the particle belonged to the quasi-projectile(QP), quasi-target (QT) or was a prompt free particle (FP) at $t_{\text {tag }}$.

In figure 3 we show the asymptotic distribution of $v_{\|}^{c m}$ calculated for every charged particle, for the two analyzed reactions.
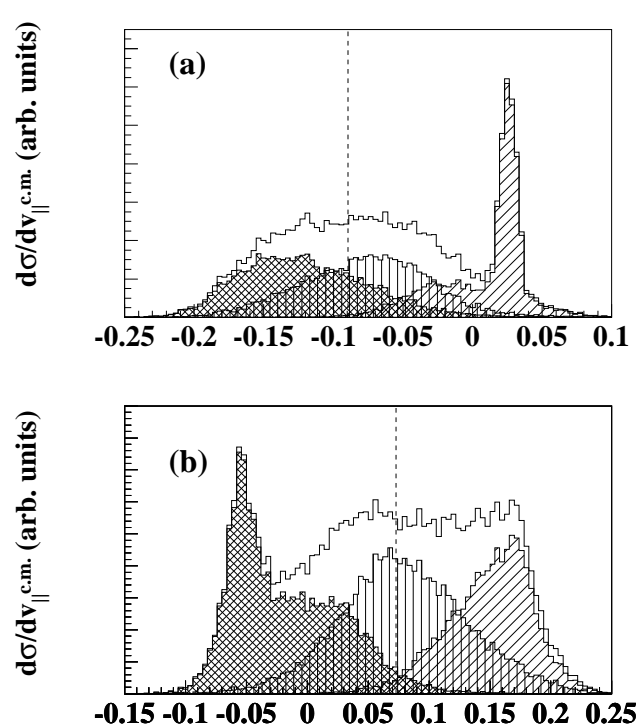

$$
\mathbf{v}_{\|} \text {c.m. (c) }
$$

FIG. 3. Normalized parallel velocity distribution, in the system center of mass, for every emitted charged particle in mid-peripheral ${ }^{58} \mathrm{Ni}+{ }^{12} \mathrm{C}$ (a), and ${ }^{58} \mathrm{Ni}+{ }^{197} \mathrm{Au}$ (b) simulated reactions. The dashed vertical line corresponds to the nucleon-nucleon velocity value. The contribution of QP, FP, and QT-tagged particles are plotted striped, shaded and grided respectively.

We considered, as in figure 2, mid-peripheral and peripheral events. In the figures, the contribution of particles that had been labelled as QT, FP, and QP at $t_{t a g}$, are distinctively shadowed.

Figure 3 (a) corresponds to the ${ }^{58} \mathrm{Ni}+{ }^{12} \mathrm{C}$ reaction $\left(2.5 \mathrm{fm} \leq b_{\text {exp }}<6.0 \mathrm{fm}\right)$. It can be seen that the particles tagged as FP were mainly emitted slightly above $v_{n n}$. Comparing with figure 2(b), it can be noticed that this emission is almost entirely composed by LCP's, dynamically expelled in the early stage of the reaction. The wide velocity distribution of the QT-tagged particles reflects the fact that the ${ }^{12} \mathrm{C}$ target is easily broken in the collision producing mainly $z \leq 3$ as byproducts. Finally it can also be seen, for the QP-tagged particles, an important contribution of intermediate mass fragments and heavier particles, aside from the presence of LCP's (see figure 2 (b)). In this case, as was remarked earlier, not only a maximum centered at the projectile-like fragment (PLF) velocity can be seen, but a second peak can also be recognized towards the mid-velocity regions.

Looking at figure 3 (b), a similar analysis can be performed for ${ }^{58} \mathrm{Ni}+{ }^{197} \mathrm{Au}$ collisions $\left(9.0 \mathrm{fm} \leq b_{\text {exp }}<12.0\right.$ $\mathrm{fm})$. In this case the FP parallel velocity distribution is centered at $v_{n n}$. Again this kind of emission is mainly composed of promptly emitted LCP's. The QP emission achieves its maximum at the PLF velocity, and presents a slight asymmetry towards the mid-velocity region. Indeed, this last feature is much more noticeable on the 
side of the heavier partner of the reaction, where an extra contribution towards the mid-rapidity, aside of the parallel velocity distribution centered around the targetlike fragment (TLF) velocity, is easily recognizable.

\section{IMF's emission-time analysis}

As we mentioned at the end of section IV, in our CMD simulations, the emission time $\left(t_{e}\right)$ of asymptotic clusters can be easily estimated. At this point, it is important to remember that the tagged-origin classification reflects spatial and velocity correlations right after the compound nucleus starts to fragment, while $t_{e}$ signals the time when a given cluster attains mass stability.

In figure 4 we used such characterization in order to plot 2-D velocity distributions, in the CM system, of IMF particles $(3 \leq Z \leq 7)$ for ${ }^{58} \mathrm{Ni}+{ }^{12} \mathrm{C}$ mid-peripheral reactions $\left(2 \mathrm{fm} \leq b_{\exp } \leq 4 \mathrm{fm}\right)$. The figures show the velocity distribution in the perpendicular plane of the collision $\left(v_{y}-v_{z}\right.$ plane), and a logarithmic scale was used for the contour levels. The contribution of QT, FP, and QP-tagged particles are shown in the first, second, and third column respectively, while particles emitted at times $t_{e}<150 \mathrm{fm} / \mathrm{c}, 150 \mathrm{fm} / \mathrm{c} \leq t_{e}<600 \mathrm{fm} / \mathrm{c}$, and $t_{e} \geq 600 \mathrm{fm} / \mathrm{c}$ after the collision are shown in the first, second and third row respectively. In the figures, we included, as reference, the mean velocities (taken over all charged particles) of QP-tagged and QT-tagged particles (solid circles).

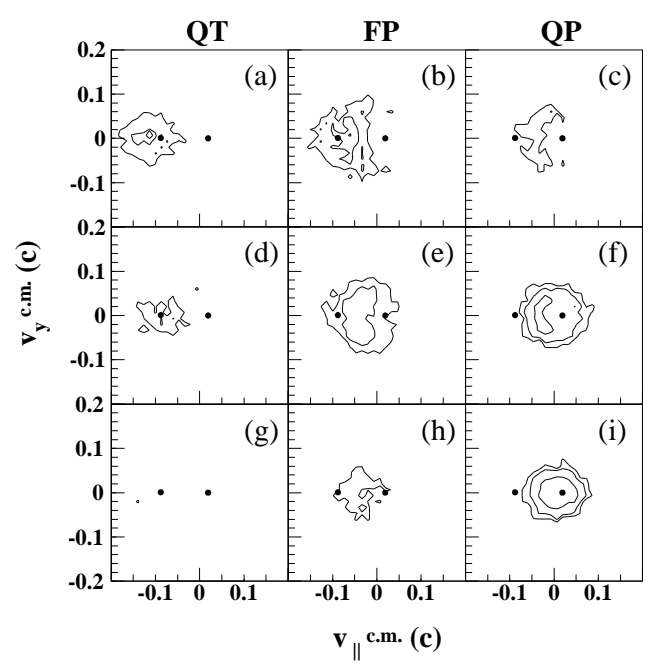

FIG. 4. Two-dimensional velocity distribution, for mid-peripheral ${ }^{58} \mathrm{Ni}+{ }^{12} \mathrm{C}$ simulated reactions. A cut perpendicular to the reaction plane is shown, i.e. $\left(v_{y}, v_{z}\right)$. The first, second, and third columns include data for QT, FP, and QP-tagged IMF's respectively. The first, second and third figure rows show the contribution of particles emitted at $t_{e}<150 \mathrm{fm} / \mathrm{c}, 150 \mathrm{fm} / \mathrm{c} \leq t_{e}<600 \mathrm{fm} / \mathrm{c}$, and $t_{e} \geq 600$ $\mathrm{fm} / \mathrm{c}$ after the beginning of the collision.

It can be seen that the QT-tagged IMF particles are emitted very early in the evolution (Figure 4 (a)). They correspond mainly to small IMF's that come from the ${ }^{12} \mathrm{C}$ breakup and, eventually, some dragged ${ }^{58} \mathrm{Ni}$ nucleons. On the other hand the FP-tagged particles (middle panels of figure 4), that were already differentiated at $t_{e}$ from the quasi-projectile and the quasi-target, seem to be emitted in a conical pattern for $t_{e}<150 \mathrm{fm} / \mathrm{c}$ reflecting the violence of the collision and the extremely asymmetric entrance channel. A second contribution of this kind of particles occurs a little bit later, $150 \mathrm{fm} / \mathrm{c} \leq t_{e}<600$ $\mathrm{fm} / \mathrm{c}$, when excited promptly ejected FP-clusters emit a second wave of FP-fragments that populate the intermediate velocity region, see Figure (e). (It is important to keep in mind, though, that the main contribution of FP-tagged particles are not IMF's but LCP's) Finally, the QP-tagged particles, shown in the third column, exhibit a very interesting behavior. The maximum IMF emission of this kind of particles occurs at intermediate times, $150 \mathrm{fm} / \mathrm{c} \leq t_{e}<600 \mathrm{fm} / \mathrm{c}$, after the collision (Figure $4(\mathrm{f}))$. A Coulomb hole in the emission pattern, that reflects the interaction between the QP-residue and the emitted IMF clusters, can easily be recognized. This kind of pattern explains the second maximum observed in the parallel velocity distribution shown in Figure 3(a), and can be considered as the signature of a dynamically induced delayed QP breakup. Note also that after this kind of delayed emission, a subsequent isotropical pattern can be observed for longer times (Figure 4 (i)). 


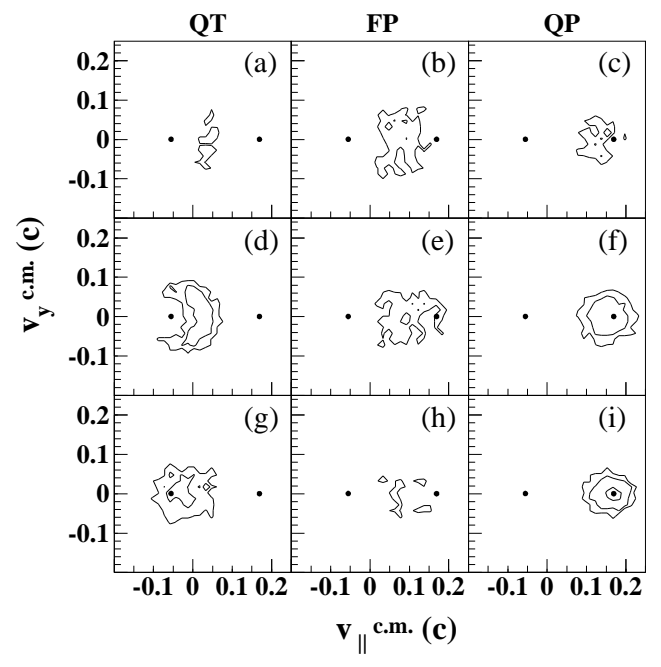

FIG. 5. Same as figure 4, but calculated for ${ }^{58} \mathrm{Ni}+{ }^{197} \mathrm{Au}$ simulated reactions.

The same analysis can be applied to the ${ }^{58} \mathrm{Ni}+{ }^{197} \mathrm{Au}$ reaction (see Figure 5), where the same kind of behavior can be observed, for very short and very long emission times (first and third row respectively). Nevertheless, the emission pattern displayed by the system at intermediate times, $150 \mathrm{fm} / \mathrm{c} \leq t_{e}<600 \mathrm{fm} / \mathrm{c}$, after the collision presents a special interest. One can observe that in this case, a delayed IMF emission pattern is recognizable mainly on the QT side of the reaction, while the QP related IMF emission is nearly isotropic at this times, in contrast to figure 4(f).

We complete the characterization of the delayed IMF emission observed in both reactions with figure 6. This figure shows, for $150 \mathrm{fm} / \mathrm{c} \leq t_{e}<600 \mathrm{fm} / \mathrm{c}$, the twodimensional velocity distributions in the reaction plane (first column), and in the perpendicular plane (second column). The first and second rows of figures correspond to ${ }^{58} \mathrm{Ni}+{ }^{12} \mathrm{C}$ and ${ }^{58} \mathrm{Ni}+{ }^{197} \mathrm{Au}$ respectively. For the sake of clarity, we did not make any distinction between QP, QT or FP-tagged contributions in this case. It can be seen that the observed delayed IMF emission occurs mainly along the QP-QT direction in both reactions. A slight deviation of this alignment is observed due to the angular momentum gained by the emission sources after the collision.
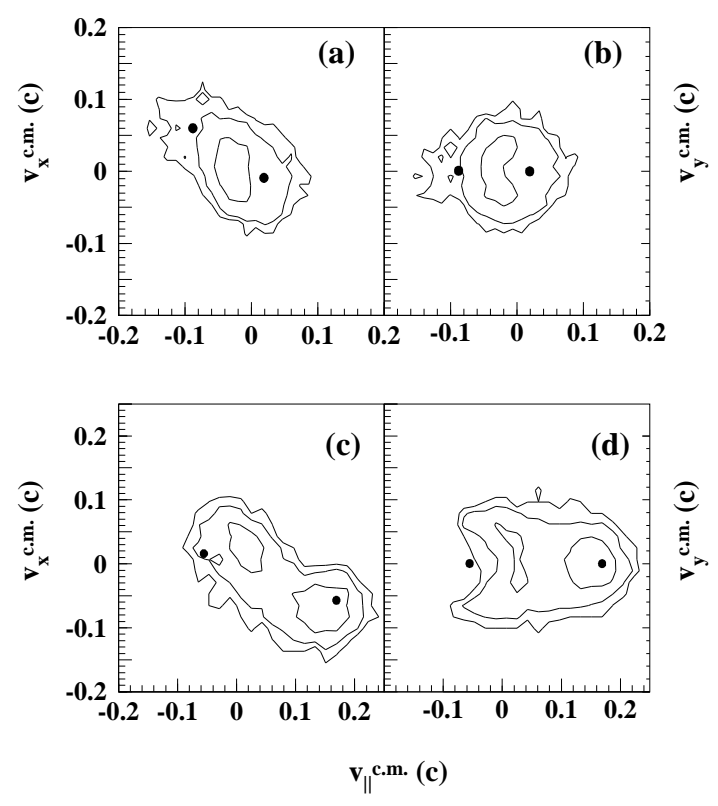

FIG. 6. Two-dimensional velocity distributions for IMF particles emitted between $t_{e}=150 \mathrm{fm} / \mathrm{c}$ and $t_{e}=600 \mathrm{fm} / \mathrm{c}$. Frames (a) and (b) correspond to mid-peripheral ${ }^{58} \mathrm{Ni}+{ }^{12} \mathrm{C}$ simulated reactions, while frames (c) and (d) to ${ }^{58} \mathrm{Ni}+{ }^{197} \mathrm{Au}$ ones. The left column shows the reaction velocity plane, $\left(v_{x}, v_{z}\right)$, while the right column shows a perpendicular cut $\left(v_{y}, v_{z}\right)$.

\section{Role of Coulomb Interactions}

In this section we will analyze the specific role played by the Coulombian interactions in the detected delayed IMF emission. To that end we study the ${ }^{58} \mathrm{Ni}+\mathrm{Au}$ reaction, comparing the behavior of the system when Coulomb interactions are not considered.

In figure 7 parallel velocity distributions are shown when Coulomb is neglected (panels (a) and (b)) and when it is considered in the simulated evolutions (panels (c) and (d)). QT emitted particles and QP emitted ones are displayed on the first and second columns respectively. The contribution of $z \geq 3$ particles is stripped, whereas IMF contribution is cross-hatched. 


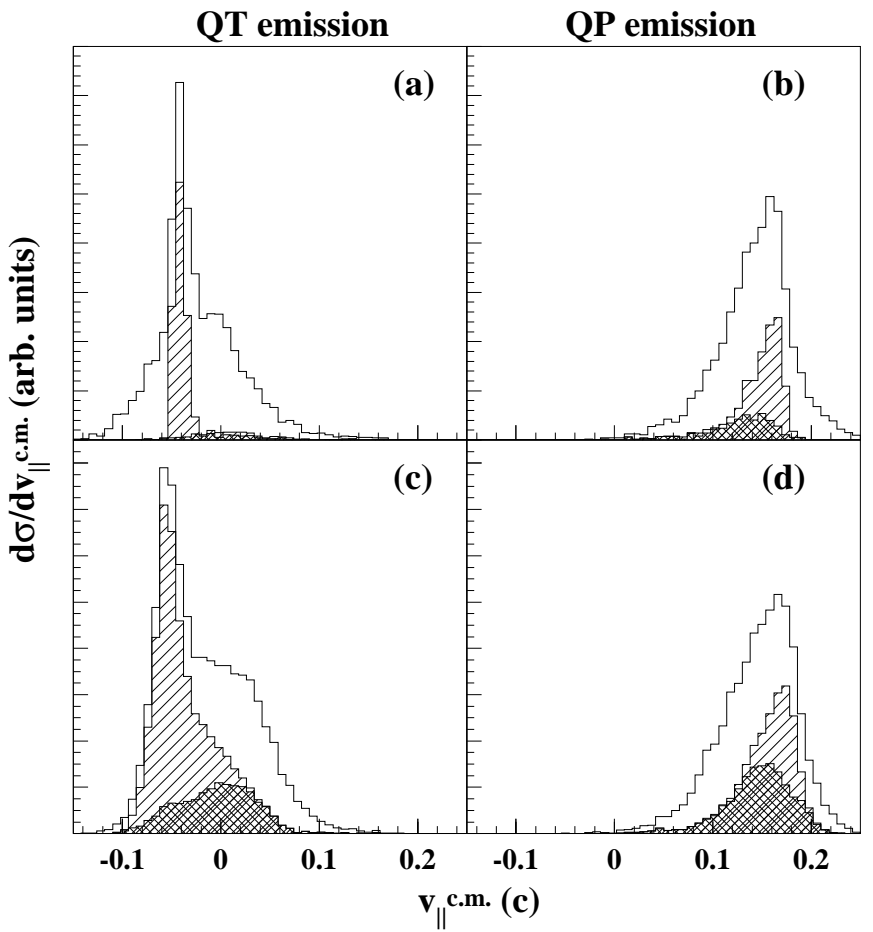

FIG. 7. Parallel velocity distribution of QT and QP emitted particles (panels (a),(c), and (b), (d)) in the simulated ${ }^{58} \mathrm{Ni}+\mathrm{Au}$ reaction. $z \geq 3$ particle contribution is stripped, whereas IMF contribution is cross-hatched. The upper panels show simulations without Colombian interaction. The lower ones include it.

It can be seen from the figure that the QT emission pattern is qualitatively affected by the presence of Coulombian interactions. In panel (a) the QT IMF production is negligeable. The few IMF present in that picture are barely attached to the quasi target, being realeased within a very short time after the projectile pass through. Without Coulomb instabilities, the dynamically induced deformations are mostly reabsorbed by the TLF.

This is not the case when electrostatic interactions are considered (panel (c)). Moreover, the overall QT emission pattern in panel (a) is rather isotropic and have lost, up to a certain degree, the memory of the entrance channel. On the contrary, the QT emission pattern when Coulombian interactions are included in the Hamiltonian (panel (c)), is severely affected by the collisional dynamics, giving place to what we called IMF aligned delayed emission.

The QP emission pattern, on the other hand, shows the same qualitative behavior with or without Coulomb. That would imply that electrostatic interactions are not the key ingredient in the QP deexcitation process.

\section{DISCUSSION}

All of these findings show the existence of two different scenarios for the origin of intermediate velocity particles. On one hand, there is an important prompt emission of LCP particles, at the highly collisional stage of the reaction. They come mainly from the overlap zone, as a consequence of nucleon-nucleon collisions that promptly eject them from the 'bulk' nuclear mean field.

On the other, a mechanism where a dynamical deformation of the heavier partner of the reaction develops can also be recognized. Eventually, this leads to the emission of the 'attached neck' followed by a Coulombian push (due to the proximity of the heavy source) that projects the emitted particles towards the intermediate velocity range. This second alternative occurs on a slower time scale and mainly involves the emission of IMF particles.

This second type of emission can be considered as a dynamically induced asymmetric fission. In fact, the study of two reactions with an inverse asymmetry in the entrance channel allowed us to reinforce the idea that this kind of delayed aligned emission has its origin in a dynamical induced shape instability that mostly affects the heavier partner of the reaction. More important deformations can be sustained by the heaviest nucleus for enough time to allow asymmetric fission processes to develop.

Coulomb effects were analyzed in the ${ }^{58} \mathrm{Ni}+\mathrm{Au}$ reaction, and no qualitative changes were observed in the emission pattern of the QP, while major ones were reported for the QT. This fact reinforces the idea that Coulomb instabilities develops mostly on the side of the biggest partner, possibly not only because of the larger charge involved, but also because the dynamical deformation settled by the reseparation dynamics is more pronounced on the biggest nuclei side.

When the two partners of the reaction reseparate, the biggest nucleus in an asymmetric reaction uses more of its surface nucleons than do the smallest one to establish the necklike bond between the two poles. Therefore, the induced shape deformation on the biggest nucleus after reseparation should be greater (the smallest nucleus has relatively not enough surface to reabsorb the neck). The neck either proceed through multiple neck ruptures before reabsortion, breaking up in the reported aligned asymmetric way, or is effectively reabsorbed by the biggest nucleus. Moreover, if we assume an equal energy sharing picture, valid for peripheral events, the $N i$ nucleus would be much hotter than the $A u$ one and could breakup by other processes before shape deformations develops. Even if some fragments could still be emitted by dynamical deformations in this case, the associated partial cross sections would be very hard to isolate. It is worth noting, though, that even if the presented picture favors the occurence of IMF delayed emission on the biggest nucleus side, nothing forbids the phenomenon to appear on a less important scale on the smallest nucleus side. 


\section{CONCLUSIONS}

In this paper, we have studied two asymmetric heavy ion reactions using the description scheme of a classical molecular dynamics model. We found that calculations are consistent with experiment, and that processes described by the model could be relevant to the experimental case.

We have made extensive use of the availability of the microscopic correlations at all times provided by this approach. Adopting the MSTE definition of fragment, the detailed knowledge of the dynamics allowed us to classify the complete set of asymptotic charged particles according to its physical situation in phase space, right after the collision (QP, QT or FP classification). Correlating this information with the emission timescale associated to each fragment, a clear distinction between different modes of particle emission in the mid-rapidity range was established. A fast emission process that mainly involve LCP, and a delayed aligned emission of IMF's, mainly coming from the heaviest partner of the reaction, were identified.

The delayed aligned emission pattern reported in this paper could possibly be compared to the statistical emission of reference [11], where a nucleus deexcite in the presence of the Coulomb field of a secondary source. Results of Ref. 11] were obtained by simulating a $\mathrm{Au}+\mathrm{Au}$ reaction at $35 \mathrm{MeV} /$ nucleon, therefore in the presence of strong Coulomb fields. According to our calculations, the same phenomenon of delayed aligned emission can be traced even in the ${ }^{58} N i+\mathrm{C}$ reaction, despite the small size of the TLF. Experimental evidences for this effect were also reported in [16]. This could indicate that dynamical effects can not be disregarded in the description of the process for light systems.

All of these findings set a reasonable framework in which recent experimental observations of particle alignment and proximity effects of the heavier partner can be understood [16. Moreover, they should be taken into account in the interpretation of other experimental observations. For example, in what concerns the geometrical and shape constraints that the observed delayed emission of IMF's impose to chemical or thermal equilibration processes in nuclear systems.

\section{ACKNOWLEDGMENT}

This work has been supported by the University of Buenos Aires (Grant No.TW98), CONICET (Grant No. PIP 4436/96), the Natural Sciences and Engineering Research Council of Canada and the Fonds pour la Formation de Chercheurs et l'Aide à la Recherche du Québec. A.Ch. acknowledges CONICET for financial support and the warm hospitality of the Laboratoire de Physique Nucléaire, Département de Physique, Université Laval (Quebec, Canada).
[1] D.R. Bowman et al., Phys. Rev. Lett. 70, 3534 (1993).

[2] C.P. Montoya et al., Phys. Rev. Lett. 73, 3070 (1994).

[3] J.F. Lecolley et al., Phys. Lett. B354, 202 (1995).

[4] J. Tõke et al., Phys. Rev. Lett. 75, 2920 (1995).

[5] J.F. Dempsey et al., Phys. Rev. C 54, 1710 (1996).

[6] Y. Larochelle et al., Phys. Rev. C 55, 1869 (1997).

[7] J. Lukasik et al., Phys. Rev. C 55, 1906 (1997).

[8] T. Lefort et al., Nucl. Phys. A662, 397 (2000).

[9] E. Plagnol et al., Phys. Rev. C 61, 014606 (2000).

[10] G. Poggi et al., Nucl. Phys. A685, 296c (2001).

[11] A. Botvina et al., Phys. Rev. C 59, 3444 (1999)

[12] Ph. Eudes and Z. Basrak, Eur. Phys. J. A 9, 207 (2000).

[13] M. Colonna, et al., Nucl. Phys. A589, 160 (1995)

[14] R. Nebauer, J. Aichelin, Nucl. Phys. A658, 67 (1999).

[15] S. Piantelli et al., arXiv:nucl-ex/0105004, (2001).

[16] L. Gingras et al., arXiv:nucl-ex/0108028, (2001).

[17] L. Gingras, M.Sc. thesis, Université Laval (1998).

[18] R.J.Lenk, T.J.Schlagel, and V.R.Pandharipande, Phys. Rev. C 42, 372 (1990).

[19] C.O.Dorso, et al., Phys. Rev. C, 60034606 (1999).

[20] A.Barrañón, et al., Rev. Mex. Fis., 45, 110, (1999).

[21] M.Belkacem et al., Phys. Rev. C, 54, 2435 (1996).

[22] D.Frenkel, B.Smit, Understanding Molecular Simulation, Academic San Diego (1996).

[23] A.Strachan and C.O.Dorso, Phys.Rev.C, 56, 995 (1997).

[24] We also took into account that the QP and QT must satisfy certain kinematical constraints, for instance: after the collision the $\mathrm{QP}(\mathrm{QT})$ candidate fragment should have a parallel velocity greater(lower) than zero in the center of mass reference system. For the mid-peripheral and peripheral reactions analyzed in this paper this procedure seemed to be suitable enough to identify reasonable quasi-sources.

[25] L. Gingras et al., in Proceedings of the XXXVIth Winter Meeting on Nuclear Physics, Bormio, Italy, edited by I.Iori, p361 (1998).

[26] L.Tassan-Got and C.Stéphan, Nucl.Phys. A524, 121 (1991). 